\title{
Peroxisomal metabolism and oxidative stress
}

Marcus Nordgren (marcus.nordgren@med.kuleuven.be)

Marc Fransen (marc.fransen@med.kuleuven.be)

Laboratory of Lipid Biochemistry and Protein Interactions, Department of Cellular and Molecular Medicine, KU Leuven, Campus Gasthuisberg, Herestraat 49 box 601, B-3000 Leuven, Belgium

\section{Highlights}

- Peroxisomes are important sites of ROS production and degradation

- Peroxisomes play a key role in the maintenance of the cellular oxidative balance

- Peroxisomes are important cellular redox signaling platforms

- Peroxisomes and mitochondria share an intricate redox-sensitive relationship

- A disturbance of peroxisomal redox homeostasis contributes to disease development 


\section{Peroxisomal metabolism and oxidative stress}

Marcus Nordgren (marcus.nordgren@med.kuleuven.be)

Marc Fransen (marc.fransen@med.kuleuven.be)

Laboratory of Lipid Biochemistry and Protein Interactions, Department of Cellular and Molecular Medicine, KU Leuven, Campus Gasthuisberg, Herestraat 49 box 601, B-3000 Leuven, Belgium

\section{Correspondence}

Dr. Marc Fransen

Katholieke Universiteit Leuven

Department of Cellular and Molecular Medicine

Laboratory of Lipid Biochemistry and Protein Interactions

Herestraat 49, box 601

B-3000 Leuven, Belgium

Phone: 003216330114

E-mail: marc.fransen@med.kuleuven.be 


\begin{abstract}
Peroxisomes are ubiquitous and multifunctional organelles that are primarily known for their role in cellular lipid metabolism. As many peroxisomal enzymes catalyze redox reactions as part of their normal function, these organelles are also increasingly recognized as potential regulators of oxidative stress-related signaling pathways. This in turn suggests that peroxisome dysfunction is not only associated with rare inborn errors of peroxisomal metabolism, but also with more common age-related diseases such as neurodegeneration, type 2 diabetes, and cancer. This review intends to provide a comprehensive picture of the complex role of mammalian peroxisomes in cellular redox metabolism. We highlight how peroxisomal metabolism may contribute to the bioavailability of important mediators of oxidative stress, with particular emphasis on reactive oxygen species. In addition, we review the biological properties of peroxisome-derived signaling messengers and discuss how these molecules may mediate various biological responses. Furthermore, we explore the emerging concepts that peroxisomes and mitochondria share an intricate redox-sensitive relationship and cooperate in cell fate decisions. This is particularly relevant to the observed demise of peroxisome function which accompanies cellular senescence, organismal aging, and agerelated diseases.
\end{abstract}

\title{
Key words
}

Peroxisome; hydrogen peroxide; lipid second messenger; mitochondrial dysfunction; agerelated diseases 


\section{Introduction}

Today, it is widely accepted that the cellular redox state is an important metabolic variable that influences many aspects of cell function, including cell survival, proliferation, and differentiation [1]. A derangement in redox homeostasis may render cells more vulnerable to oxidative stress, a condition in which the production of reactive oxygen and/or nitrogen species (ROS/RNS) ${ }^{1}$ overwhelms the capacity of the antioxidant defense and repair mechanisms [2]. Major cellular sources of ROS/RNS encompass the electron transport chain in mitochondria, the Ero1 and cytochrome P-450 enzymes in the endoplasmic reticulum (ER), the NADPH oxidases at the plasma membrane, the flavin oxidases inside peroxisomes, and the nitric oxide synthases (NOSs) which show different subcellular localizations [3]. Natural antioxidant systems include various enzymes (e.g. superoxide dismutase, glutathione peroxidase, and catalase) and non-enzymatic metabolites (e.g. glutathione and ascorbic acid) [3]. Depending on the type of ROS/RNS, their concentration and localization, and their kinetics of production and elimination, these small reactive molecules may propagate downstream signaling events or cause oxidative damage to biomolecules [4]. As such, it is not surprising that both acute and sustained alterations in the redox state can contribute to the mechanisms of cellular aging and age-related diseases [4]. In the following sections of this review, we focus on the role of peroxisomes in oxidative stress- and antioxidant defenserelated pathways in mammals (Fig. 1).

\section{Peroxisomes are important sites of ROS production and degradation}

As indicated by their name, peroxisomes play a central role in the cellular metabolism of hydrogen peroxide $\left(\mathrm{H}_{2} \mathrm{O}_{2}\right)$ [5]. This is perhaps best illustrated by the fact that these organelles harbor copious amounts of enzymes that can produce or degrade this molecule. The best known ones are the $\mathrm{H}_{2} \mathrm{O}_{2}$-producing flavin-containing oxidases and catalase, a $\mathrm{H}_{2} \mathrm{O}_{2}$ decomposing enzyme [6]. Peroxisomes also contain enzymes that generate superoxide $\left(\mathrm{O}_{2}{ }^{\circ}\right)$ (e.g. xanthine oxidase) or nitric oxide (NO') (e.g. xanthine oxidase and NOS2, the inducible form of nitric oxide synthase) as part of their normal catalytic activity [6]. In addition, as NO

\footnotetext{
${ }^{1}$ Abbreviations: AA, arachidonic acid; DHA, docosahexaenoic acid; ER, endoplasmic reticulum; GSH, reduced glutathione; GSSH, oxidized glutathione; LONP2, peroxisomal Lon protease; NOSs, nitric oxide synthases; PUFAs, polyunsaturated fatty acids; RNS, reactive nitrogen species; ROS, reactive oxygen species; UOX, urate oxidase; VLCFAs, very-long-chain fatty acids; X-ALD, Xlinked adrenoleukodystrophy.
} 
may rapidly combine with $\mathrm{O}_{2}{ }^{--}$to form peroxynitrite $\left(\mathrm{ONOO}^{-}\right.$) [7], and $\mathrm{H}_{2} \mathrm{O}_{2}$ may give rise to hydroxyl radicals $\left({ }^{\circ} \mathrm{OH}\right)$ through the Fenton reaction [8], it is very likely that these organelles also have the potential to act as a source of these ROS/RNS species. Importantly, since $\mathrm{ONOO}^{-}$and ${ }^{\circ} \mathrm{OH}$ are highly unstable, they can cause direct oxidative biomolecular damage, such as lipid peroxidation [9]. In this context, it is essential to mention that peroxisomes also contain antioxidant enzymes that can degrade $\mathrm{O}_{2}^{-{ }^{-}}$(e.g. superoxide dismutase 1), $\mathrm{ONOO}^{-}$(e.g. peroxiredoxin 5), epoxides (e.g. epoxide hydrolase 2), and lipid peroxides (e.g. peroxiredoxin 5 and glutathione S-transferase kappa). For a detailed description of these and other peroxisomal pro- and antioxidant enzymes, we refer the reader to other comprehensive reviews covering this topic $[6,10,11]$.

As already mentioned in the introduction, the major non-enzymatic cellular redox buffer systems rely on the antioxidants glutathione and ascorbic acid. Glutathione ( $\gamma$-glutamylcysteinyl-glycine) is a tripeptide that, within cells, can exist in reduced (GSH) and oxidized (GSSG) states [3]. Ascorbic acid, also known as vitamin C, is an essential nutrient in human diets that functions as cofactor for a number of enzymes and is capable of scavenging various ROS/RNS. Although it is well documented that plant peroxisomes contain a functional ascorbate-glutathione cycle [12], relatively little is known about the network of nonenzymatic antioxidants inside mammalian peroxisomes. Nonetheless, there is some indirect evidence that GSH may freely diffuse from the cytosol into peroxisomes via PXMP2, a nonselective pore-forming peroxisomal membrane protein with an upper molecular size limit of 300-600 Da [13]. However, it remains to be determined how GSSG can be reduced inside the peroxisomal matrix or exported back into the cytosol. In addition, although it has been demonstrated that ascorbic acid functions as a cofactor for phytanoyl-CoA 2-hydroxylase in the peroxisomal matrix [14], it remains unclear whether or not this vitamin also displays antioxidant properties in this subcellular compartment. Indeed, a recent study from our laboratory has shown that the cultivation of mouse embryonic fibroblasts in media containing ascorbic acid actually led to an increased redox state of the peroxisomal matrix [15]. This may be explained by the facts that (i) peroxisomes contain relatively large amounts of heme- and non-heme iron-containing enzymes [16], and (ii) ascorbic acid can generate hydroxyl and alkoxyl radicals in the presence of free transition metals [17].

Finally, peroxisomes also harbor several proteases whose functions may be linked to peroxisomal ROS-production. One such enzyme is peroxisomal Lon peptidase (LONP2), an 
enzyme that - among other functions - is implicated in the degradation of dysfunctional and/or excessive matrix proteins [18,19]. Indeed, in a recent study in Penicillium chrysogenum, it was shown that LONP2 selectively degrades oxidatively damaged proteins in the peroxisomal matrix, and that an inactivation of this protein enhances cellular oxidative stress [20]. In addition, it has been demonstrated that this protease is involved in the removal of excess peroxisomal $\beta$-oxidation enzymes upon removal of proliferation stimuli [18]. This observation is in line with a recent study showing that LONP2 proteolytically regulates peroxisomal fatty acid $\beta$-oxidation [19]. Taken together, these findings indicate that LONP2 may act as a significant coordinator of metabolism-related ROS generation within these organelles. Lastly, also insulin-degrading enzyme, another peroxisomal protease, has been shown to be capable of degrading oxidized proteins [21]. In summary, these proteases are likely to influence peroxisomal ROS production by regulating the quantity and quality of the organellar matrix protein content.

\section{Peroxisomes play a key role in the maintenance of cellular oxidative balance}

Peroxisomes house many enzymes that produce or degrade ROS/RNS (see Section 2). As such, they have the intrinsic properties to act as modulators of cellular oxidative balance. However, as most ROS/RNS (e.g. $\mathrm{O}_{2}{ }^{-}, \mathrm{H}_{2} \mathrm{O}_{2},{ }^{\circ} \mathrm{OH}$, and $\mathrm{ONOO}^{-}$) cannot freely diffuse across a lipid bilayer [22], a conditio sine qua non for being integrated into the cellular redox regulatory network is that the peroxisomal membrane does not constitute a hurdle for the diffusion of redox molecules from one compartment to the other. Importantly, this criterion is most likely fulfilled, given that peroxisomes in mammals contain a non-selective membrane pore large enough to accommodate the diffusion of virtually all types of ROS/RNS that can be generated or metabolized inside the organelle (see Section 2). Nevertheless, in this context, it is worth adding that - despite the high content of catalase in the peroxisomal matrix peroxisomes in metabolically active rat liver slices have been reported to be inefficient detoxifiers of external $\mathrm{H}_{2} \mathrm{O}_{2}$, and that the highly packed matrix within the organelle itself seems to act as diffusion barrier [23]. Interestingly, the same study also showed that the tubular structures in crystalloid cores of urate oxidase (UOX) in these peroxisomes serve as exhaust conduits that release UOX-derived $\mathrm{H}_{2} \mathrm{O}_{2}$ directly into the extraperoxisomal space.

Given that peroxisomes play a central role in cellular lipid metabolism, they may also help protect cells from oxidative stress by actively maintaining the optimal membrane lipid 
composition (Fig. 1) [24]. In this context, it is essential to note that peroxisomes harbor enzymes that are involved in the metabolism of very-long-chain fatty acids (VLCFAs) and the biosynthesis of docosahexaenoic acid (DHA) and plasmalogens [25]. As these lipophilic molecules are able to insert into cellular lipid bilayers, it is very likely that changes in their abundance also alter membrane structure, fluidity, and function. In addition, higher concentrations of polyunsaturated fatty acids (PUFAs) are thought to sensitize cells to oxidative stress due to an increased likelihood of lipid peroxidation [26], and plasmalogens are not only structural components of cell membranes but may also function as physiological antioxidants with their vinyl ether functionality serving as sacrificial trap for free radicals [27].

Currently, there is plenty of evidence that disturbances in peroxisomal (redox) metabolism sensitize cells to oxidative stress [11]. For example, mouse embryonic fibroblasts lacking glyceronephosphate O-acyltransferase, a peroxisomal enzyme catalyzing the first step in plasmalogen biosynthesis, are more susceptible to 2,2'-azobis(2-methylpropionamidine) dihydrochloride-induced oxidative stress [28]; fibroblasts from patients with peroxisome biogenesis disorders are more sensitive to UV light-induced oxidative stress [29]; cultured cerebellar neurons from peroxisome-deficient mice display increased oxidative stress and apoptosis [30]; and inhibition of catalase activity causes damage to proteins and DNA, increases mitochondrial ROS production, and impairs cell growth [15,31,32]. On the other hand, it is also important to take into account that cellular oxidative stress may affect peroxisome morphology [33,34], motility [35], and function (e.g. by reducing the import kinetics of matrix proteins) [15,36]. In this context, it is noteworthy that (i) a recent study has shown that valosin-containing protein can sense $\mathrm{H}_{2} \mathrm{O}_{2}$ via a highly reactive cysteine residue, and regulate $\mathrm{H}_{2} \mathrm{O}_{2}$ levels in the cytosol by affecting the retention time of (newly-synthesized) catalase within this subcellular compartment [37], (ii) we found that also Pex5p, the cycling import receptor for peroxisomal matrix proteins, is a redox-sensitive protein (our unpublished results), and (iii) others have reported that, in Arabidopsis thaliana (but most likely also in other organisms, including mammals), the activity of peroxisomal 3-ketoacyl-Coa thiolase is controlled by a redox-sensitive switch that may regulate peroxisomal $\beta$-oxidation (and hence peroxisomal $\mathrm{H}_{2} \mathrm{O}_{2}$ production) [38]. In summary, these and other findings clearly show that peroxisomal metabolism and cellular oxidative balance are intimately interconnected. However, the mechanisms by which peroxisomes may act as redox signaling platforms have only recently begun to emerge. 


\section{Peroxisomes are important cellular redox signaling platforms}

Over the last years, it has become increasingly clear that changes in peroxisomal metabolism may have a profound impact on cellular processes by modulating the composition and concentration of specific lipids and (redox-derived) signaling mediators (Fig. 1) [39,40]. Here, it is essential to bear in mind that (i) any change in the production, constitution and/or localization of (phospho)lipids may have profound effects on cellular signaling cascades [41], and (ii) virtually all stress stimuli trigger changes in lipid composition [42]. In addition, it is well known that the localization and activity of many proteins (e.g. kinases, phosphatases, and transcription factors) are reversibly controlled by the oxidation state of specific cysteine thiols [43]. In the following paragraphs, we review the biological properties of peroxisome-derived signaling messengers and discuss how these molecules may mediate various biological responses.

Peroxisomes are relevant sources of different types of ROS/RNS (see Section 1). Of these, $\mathrm{H}_{2} \mathrm{O}_{2}$ and $\mathrm{NO}^{\circ}$ are, due to their life time and potential diffusion distance, considered the most favorable ones to act as signaling molecules $[44,45]$. The precise cellular responses to peroxisome-derived $\mathrm{H}_{2} \mathrm{O}_{2}$ and $\mathrm{NO}^{\circ}$ are not yet well understood. However, in analogy with other systems, one may anticipate that these responses are concentration dependent in that excess amounts of peroxisomal $\mathrm{H}_{2} \mathrm{O}_{2}$ and $\mathrm{NO}^{\circ}$ can be expected to be cytotoxic while low concentrations may mediate various responses such as changes in gene expression and cell growth. In this context, it is relevant to know that, at low (physiological) concentrations, both $\mathrm{H}_{2} \mathrm{O}_{2}$ and $\mathrm{NO}^{\circ}$ have preferred biological targets. For example, $\mathrm{H}_{2} \mathrm{O}_{2}$ can - directly or with the help of thiol peroxidases or sulfhydryl oxidases - oxidize proteins by converting thiol groups of reactive cysteines to sulphenic acids or disulfide bonds $[45,46]$; and $\mathrm{NO}^{\bullet}$ is capable of posttranslationally modifying proteins by converting thiol groups of reactive cysteines to nitrosothiols [47]. Importantly, these modifications are thought to change the function of a broad spectrum of proteins.

In support of these ideas, it has been shown that (i) inhibition of catalase activity by 3 aminotriazole increases the cellular protein disulfide content by $20 \%$ [48]; (ii) overexpression of catalase sensitizes cells (and animals) to certain types of stressors by dampening $\mathrm{H}_{2} \mathrm{O}_{2-}$ mediated signaling pathways [49,50]; (iii) overexpression of acyl-CoA oxidase $1, \mathrm{a}_{2} \mathrm{O}_{2}$ - 
producing enzyme of the peroxisomal fatty acid $\beta$-oxidation pathway, can activate NF- $\mathrm{kB}$, a redox-sensitive transcription factor that regulates various inflammatory and cell cycle regulatory genes, in a substrate concentration-dependent manner [51]; and (iv) excess peroxisome-derived $\mathrm{H}_{2} \mathrm{O}_{2}$ functions as an important mediator of lipotoxicity in insulinproducing cells [52]. In addition, although the intraperoxisomal localization and activity of $\mathrm{NO}^{\circ}$-producing enzymes in mammals remains enigmatic [53,54], there is experimental proof that various peroxisomal proteins (e.g. catalase, 3-ketoacyl-CoA thiolase B, Pex11p $\alpha, \ldots$ ) can be selectively S-nitrosylated, at least in some mouse tissues [55]. Nevertheless, the physiological and pathophysiological roles of $\mathrm{NO}^{\circ}$ production and action inside mammalian peroxisomes remain to be elucidated. Here, two notable hypotheses have been considered regarding the possible role of NOS2 inside peroxisomes. The first hypothesis proposes that peroxisomal NOS2 may function to modulate the organellar enzyme activities [54]. This postulate, which was predominantly based on the finding that the appearance of NOS2 inside peroxisomes is associated with a decrease in catalase activity [54], is in line with the recent observation that various peroxisomal proteins, including catalase, can be S-nitrosylated [55]. The second hypothesis suggests that NOS2 localizes to peroxisomes as a protective mechanism to remove catalytically incompetent variants of this enzyme [56]. Here it is essential to note that NOS2, in its monomeric form or in the absence of adequate substrate, can produce $\mathrm{O}_{2}{ }^{-}[56]$.

Unfortunately, virtually nothing is known regarding the specific effects and physiological functions of other peroxisomal ROS/RNS species, such as $\mathrm{O}_{2}{ }^{-},{ }^{\circ} \mathrm{OH}$, and $\mathrm{ONOO}^{-}$. However, here it should be mentioned that (i) $\mathrm{O}_{2}{ }^{-}$is considered to be a major precursor of $\mathrm{H}_{2} \mathrm{O}_{2}$ rather than a direct participant in signaling [45], (ii) ${ }^{\circ} \mathrm{OH}$ lacks any specificity as it reacts with almost any organic molecule it encounters [45], and (iii) $\mathrm{ONOO}^{-}$is a short-lived strong oxidant that can oxidize protein-associated thiol groups, nitrate tyrosine residues on proteins, and initiate lipid peroxidation [57]. Finally, there is currently no evidence that mammalian peroxisomes can serve as a potential source of S-nitrosoglutathione, a physiological NO carrier.

Another set of peroxisome-related signaling molecules that can conceivably regulate cellular processes include plasmalogens, PUFAs, and sphingolipids. Plasmalogens may serve as precursors of biologically active lipid mediators, as (i) they often contain arachidonic acid (AA) or DHA at the sn-2 position of the glycerol moiety, and (ii) upon release by the action of 
phospholipase 2A, these PUFAs can be metabolized to second messengers that modulate inflammatory responses (e.g. prostaglandins, thromboxanes, leukotrienes, docosanoids, ...) [58]. In addition, AA, DHA and other PUFAs are known to be major targets for lipid peroxidation [59], and 4-hydroxy-2-nonenal - one of the major end products of this process can modulate both cytoprotective and cytotoxic signal transduction pathways [60]. Finally, as brains and fibroblasts of mice and patients with peroxisomal disorders contain increased levels of C26:1/0-ceramide [61], changes in peroxisomal metabolism may also exert an influence on the physiological responses mediated by the sphingolipid class of bioactive lipids. Note that members of this class of lipids act as important messengers for signaling events that lead to cell proliferation, differentiation, and senescence [42]. In summary, the data presented in this section strongly support the idea that peroxisomes actively contribute to trans-compartmental lipid and ROS signaling in mammalian cells.

\section{Peroxisomes and mitochondria share an intricate redox-sensitive relationship}

Over time, it has become increasingly clear that peroxisomes extensively cooperate with other organelles, such as mitochondria [62,63], the ER [40], and lipid droplets [64], to optimally perform their cellular tasks. Peroxisomes and mitochondria are notably interconnected in that they (i) collaborate at different levels to maintain various metabolic and signaling pathways $[25,62,63]$, (ii) share several crucial components of their organellar fission machineries $[65,66]$, and (iii) display a redox-sensitive relationship [11]. Regarding the latter, it has been shown that excessive ROS-generation in peroxisomes increases the mitochondrial redox state and triggers mitochondrial fragmentation with subsequent (apoptotic) cell death [15; our unpublished observations]. Here it is important to mention that the relationship between mitochondrial oxidative stress and cell death is well established [67], and that the potential role of peroxisomes in cell death pathways is just beginning to emerge (Fig. 1). However, in this context, it should be stressed that peroxisome dysfunction also has a profound impact on mitochondrial function. For example, Pex5p knockout mice possess increased levels of mitochondria, which show structural abnormalities and alterations in the expression and activities of respiratory chain complexes [68,69]. In addition, mitochondrial oxidative phosphorylation seems also to be impaired in X-linked adrenoleukodystrophy (X-ALD), the most common peroxisomal disorder [70]. Importantly, this study as well as several other reports link disturbances in peroxisomal redox metabolism to mitochondrial oxidative stress. For example, it has been shown that a reduction in catalase activity contributes to oxidative 
stress-dependent mitochondrial dysfunction $[31,71,72]$, and that mitochondrial redox balance demonstrate that changes in peroxisomal metabolism have a profound impact on mitochondrial functions.

\section{A disturbance of peroxisomal redox homeostasis contributes to disease development}

During the last decades, it has become increasingly clear that peroxisome dysfunction is not restricted to inherited peroxisomal diseases, but also to disease processes associated with aging [24]. These pathological processes include oxidative stress, cellular dysfunction, and inflammation. In the following paragraphs, each of these themes is discussed in more depth. When relevant, this will be illustrated with examples. However, for a detailed overview on the role of peroxisomes in specific age-related disorders, we refer to another recent review [24].

As peroxisomes are important sites of ROS/RNS production and degradation, it is no surprise that these organelles have garnered increasing attention for their potential role in oxidative stress-related signaling pathways and pathologies [11]. A hypothesis gaining prominence is that low levels of peroxisomal ROS/RNS act as signaling molecules that promote cell proliferation and cell survival (= concept of peroxihormesis [24]), and that a profound disturbance of peroxisomal metabolism triggers signaling/communication events that ultimately result in the activation of (mitochondrial) cell death pathways (Fig. 1) [73]. In this context, it is interesting to note that (i) a preservation of peroxisome function exerts a protective effect against ROS-induced apoptosis during acute kidney injury [74], (ii) peroxisomal ROS metabolism plays a key role in the regulation of the hypothalamic melanocortin tone and food intake in diet-induced obesity [75], (iii) the pancreatic $\beta$-cell lipotoxicity induced by free fatty acids is caused by $\mathrm{H}_{2} \mathrm{O}_{2}$ produced through peroxisomal $\beta$ oxidation [52], (iv) a deficiency in catalase activity accelerates diabetic renal injury through peroxisomal dysfunction [72], and (v) disturbances in peroxisome function result in enhanced neuronal cell death [76]. Regarding the latter observation, it is important to mention that longlived neurons are particularly vulnerable to the effects of ROS/RNS due to their high demand for oxygen and abundance of peroxidizable lipids [77].

Increasing evidence suggests that disturbances in peroxisomal metabolism do also play an important role in the accumulation of oxidation-mediated cellular damage and aging [78]. 
This can be well illustrated by the observations that (i) catalase levels and activity drop with age, at least in rats $[79,80]$, (ii) hypocatalasemic fibroblasts accumulate $\mathrm{H}_{2} \mathrm{O}_{2}$, are oxidatively damaged, and display age-associated pathologies [81], and (iii) these phenotypes can be reversed by forced overexpression of catalase-SKL, a catalase derivative with enhanced peroxisome targeting efficiency [31]. At first sight, these findings may seem surprising given that cellular aging is widely considered to be driven by excess mitochondrial ROS production. However, in the meantime, there is sufficient evidence to foster the idea that peroxisomes can act as upstream initiators of mitochondrial ROS signaling pathways [24,32,82]. Importantly, the redox signaling pathways between peroxisomes and mitochondria remain to be elucidated. In summary, these observations and the recent finding that cellular senescence is causally implicated in generating age-related phenotypes [83], suggest that alterations in peroxisome function most likely play a more prominent role in the human aging process than we currently think [24]. However, as cellular oxidative stress has also been implicated as a causative factor in the development of peroxisome dysfunction (e.g. by affecting the import kinetics of peroxisomal matrix proteins $[15,36,37])$, much work remains to be done to gain full understanding of the cause-and-effect relationships between peroxisome dysfunction, oxidation-mediated cellular damage, and disease development.

Oxidative stress and inflammation are strongly related to each other, and - as such - it may not be surprising that a growing body of evidence emphasizes the potential role of peroxisomes in inflammatory processes [84]. For example, it has become apparent that (i) peroxisomes have the potential to regulate the bioavailability of important inflammatory mediators (e.g. $\mathrm{H}_{2} \mathrm{O}_{2}$, $\mathrm{NO}^{\circ}$, prostaglandins, leukotrienes, ...) $[85,86]$, and (ii) peroxisome inactivity can trigger fast neuroinflammatory reactions $[87,88]$. In addition, there are indications that inflammatory mediators such as proinflammatory cytokines can downregulate peroxisome function [85]. This in turn may lead to an accumulation of VLCFAs, and - as lipid derivatives with an abnormally high proportion of VLCFAs have been reported to trigger inflammatory responses and demyelination [89] - this may initiate a perpetual inflammatory cascade $[85,87]$.

\section{Conclusions and future directions}

Over the years, a considerable amount of evidence has been collected that disturbances in peroxisomal metabolism can have significant consequences for human health. However, at 
present, it is still unclear the extent to which defects in peroxisomal metabolism lead to cellular and organismal pathologies. An attractive hypothesis put forth is that peroxisomes mediate developmental decisions by modulating the cellular composition and concentration of specific lipids and (redox-derived) signaling mediators (Fig. 1) [40,73]. Specifically, it is thought that - when peroxisome activity is optimal - the organelle activates cytoprotective and anti-aging mechanisms, while - under non-optimal conditions - the organelle becomes a signaling platform governing pro-aging processes [40,73]. An intriguing and still open question is how peroxisomes contribute to stress responses and metabolic pathways that potentially impinge on the aging process and for example cause neurological decline. Gaining a better insight into these issues requires more data about (i) the identity of proximal targets for peroxisomal ROS/RNS, (ii) the downstream signaling pathways regulated by these factors, and (iii) the molecular mechanisms underlying the stress-related communication events between peroxisomes and mitochondria. Although such experiments will be technically challenging, being successful is the only key to figure out the precise role of peroxisomes in the initiation and progression of oxidative stress-related diseases.

\section{Acknowledgements}

Marcus Nordgren is a recipient of a FLOF fellowship from the Department of Cellular and Molecular Medicine at the KU Leuven. Marc Fransen is supported by grants from the 'Fonds voor Wetenschappelijk Onderzoek-Vlaanderen (Onderzoeksproject G.0754.09)' and the 'Bijzonder Onderzoeksfonds van de KU Leuven (OT/09/045)'.

\section{References}

[1] R. Ivarsson, R. Quintens, S. Dejonghe, K. Tsukamoto, P. in 't Veld, E. Renström, F.C. Schuit, Redox control of exocytosis: regulatory role of NADPH, thioredoxin, and glutaredoxin, Diabetes 54 (2005) 2132-2142.

[2] A.B. Salmon, A. Richardson, V.I. Pérez, Update on the oxidative stress theory of aging: does oxidative stress play a role in aging or healthy aging? Free Radic. Biol. Med. 48 (2010) 642-655.

[3] C. Nathan, A. Ding, SnapShot: reactive oxygen intermediates (ROI), Cell 140 (2010) 951-951. 
[4] M.D. Temple, G.G. Perrone, I.W. Dawes, Complex cellular responses to reactive oxygen species, Trends Cell Biol. 15 (2005) 319-326.

[5] C. de Duve, P. Baudhuin, Peroxisomes (microbodies and related particles), Physiol. Rev. 46 (1966) 323-357.

[6] V.D. Antonenkov, S. Grunau, S. Ohlmeier, J.K. Hiltunen, Peroxisomes are oxidative organelles, Antioxid. Redox Signal. 13 (2010) 525-537.

[7] P. Pacher, J.S. Beckman, L. Liaudet, Nitric oxide and peroxynitrite in health and disease, Physiol. Rev. 87 (2007) 315-424.

[8] S.K. Powers, M.J. Jackson, Exercise-induced oxidative stress: cellular mechanisms and impact on muscle force production, Physiol. Rev. 88 (2008) 1243-1276.

[9] H. Yin, L. Xu, N.A. Porter, Free radical lipid peroxidation: mechanisms and analysis, Chem. Rev. 111 (2011) 5944-5972.

[10] N.A. Bonekamp, A. Völkl, H.D. Fahimi, M. Schrader, Reactive oxygen species and peroxisomes: struggling for balance, Biofactors 35 (2009) 346-355.

[11] M. Fransen, M. Nordgren, B. Wang, O. Apanasets, Role of peroxisomes in ROS/RNSmetabolism: implications for human disease, Biochim. Biophys. Acta 1822 (2012) 1363-1373.

[12] L.A. del Río, L.M. Sandalio, F.J. Corpas, J.M. Palma, J.B. Barroso, Reactive oxygen species and reactive nitrogen species in peroxisomes. Production, scavenging, and role in cell signaling, Plant Physiol. 141 (2006) 330-335.

[13] A. Rokka, V.D. Antonenkov, R. Soininen, H.L. Immonen, P.L. Pirilä, U. Bergmann, R.T. Sormunen, M. Weckström, R. Benz, J.K. Hiltunen, Pxmp2 is a channel-forming protein in mammalian peroxisomal membrane, PLoS One 4 (2009) e5090.

[14] K. Croes, V. Foulon, M. Casteels, P.P. Van Veldhoven, G.P. Mannaerts, PhytanoylCoA hydroxylase: recognition of 3-methyl-branched acyl-CoAs and requirement for GTP or ATP and $\operatorname{Mg}(2+)$ in addition to its known hydroxylation cofactors, J. Lipid Res. 41 (2000) 629-636.

[15] O. Ivashchenko, P.P. Van Veldhoven, C. Brees, Y.S. Ho, S.R. Terlecky, M. Fransen, Intraperoxisomal redox balance in mammalian cells: oxidative stress and interorganellar cross-talk, Mol. Biol. Cell 22 (2011) 1440-1451.

[16] T.B. Dansen, K.W. Wirtz, The peroxisome in oxidative stress, IUBMB Life 51 (2001) 223-230. 
[17] M.C. Putchala, P. Ramani, H.J. Sherlin, P. Premkumar, A. Natesan, Ascorbic acid and its pro-oxidant activity as a therapy for tumours of oral cavity - A systematic review, Arch. Oral Biol. 58 (2013) 563-574.

[18] S. Yokota, C.M. Haraguchi, T. Oda, Induction of peroxisomal Lon protease in rat liver after di-(2-ethylhexyl)phthalate treatment, Histochem. Cell Biol. 129 (2008) 73-83.

[19] K. Okumoto, Y. Kametani, Y. Fujiki, Two proteases, trypsin domain-containing 1 (Tysnd1) and peroxisomal Lon protease (PsLon), cooperatively regulate fatty acid $\beta$ oxidation in peroxisomal matrix, J. Biol. Chem. 286 (2011) 44367-44379.

[20] M. Bartoszewska, C. Williams, A. Kikhney, Ł Opaliński, C.W. van Roermund, R. de Boer, M. Veenhuis, I.J. van der Klei, Peroxisomal proteostasis involves a Lon family protein that functions as protease and chaperone, J. Biol. Chem. 287 (2012) 2738027395.

[21] M. Morita, I.V. Kurochkin, K. Motojima, S. Goto, T. Takano, S. Okamura, R. Sato, S. Yokota, T. Imanaka, Insulin-degrading enzyme exists inside of rat liver peroxisomes and degrades oxidized proteins, Cell Struct. Funct. 25 (2000) 309-315.

[22] G.P. Bienert, J.K. Schjoerring, T.P. Jahn, Membrane transport of hydrogen peroxide, Biochim. Biophys. Acta 1758 (2006) 994-1003.

[23] R. Fritz, J. Bol, U. Hebling, S. Angermüller, A. Völkl, H.D. Fahimi, S. Mueller, Compartment-dependent management of hydrogen peroxide by peroxisomes, Free Radic. Biol. Med. 42 (2007) 1119-1129.

[24] M. Fransen, M. Nordgren, B. Wang, O. Apanasets, P. Van Veldhoven, Aging, agerelated diseases and peroxisomes, Subcell. Biochem. 69 (2013) 45-65.

[25] P.P. Van Veldhoven, Biochemistry and genetics of inherited disorders of peroxisomal fatty acid metabolism, J. Lipid Res. 51 (2010) 2863-2895.

[26] M. Perluigi, R. Coccia, D.A. Butterfield, 4-Hydroxy-2-nonenal, a reactive product of lipid peroxidation, and neurodegenerative diseases: a toxic combination illuminated by redox proteomics studies, Antioxid. Redox Signal. 17 (2012) 1590-1609.

[27] S. Wallner, G. Schmitz, Plasmalogens the neglected regulatory and scavenging lipid species, Chem. Phys. Lipids 164 (2011) 573-589.

[28] A. Brodde, A. Teigler, B. Brugger, W.D. Lehmann, F. Wieland, J. Berger, W.W. Just, Impaired neurotransmission in ether lipid-deficient nerve terminals, Hum. Mol. Genet. 21 (2012) 2713-2724. 
[29] G. Hoefler, E. Paschke, S. Hoefler, A.B. Moser, H.W. Moser, Photosensitized killing of cultured fibroblasts from patients with peroxisomal disorders due to pyrene fatty acidmediated ultraviolet damage, J. Clin. Invest. 88 (1991) 1873-1879.

[30] C.C. Müller, T.H. Nguyen, B. Ahlemeyer, M. Meshram, N. Santrampurwala, S. Cao, P. Sharp, P.B. Fietz, E. Baumgart-Vogt, D.I. Crane, PEX13 deficiency in mouse brain as a model of Zellweger syndrome: abnormal cerebellum formation, reactive gliosis and oxidative stress, Dis. Model Mech. 4 (2011) 104-119.

[31] J.I. Koepke, K.A. Nakrieko, C.S. Wood, K.K. Boucher, L.J. Terlecky, P.A. Walton, S.R. Terlecky, Restoration of peroxisomal catalase import in a model of human cellular aging, Traffic 8 (2007) 1590-1600.

[32] P.A. Walton, M. Pizzitelli, Effects of peroxisomal catalase inhibition on mitochondrial function, Front. Physiol. 3 (2012). doi: 10.3389/fphys.2012.00108.

[33] M. Schrader, R. Wodopia, H.D. Fahimi, Induction of tubular peroxisomes by UV irradiation and reactive oxygen species in HepG2 cells, J. Histochem. Cytochem. 47 (1999) 1141-1148.

[34] A.M. Sinclair, C.P. Trobacher, N. Mathur, J.S. Greenwood, J. Mathur, Peroxule extension over ER-defined paths constitutes a rapid subcellular response to hydroxyl stress, Plant J. 59 (2009) 231-242.

[35] M. Rodríguez-Serrano, M.C. Romero-Puertas, I. Sparkes, C. Hawes, L.A. del Río, L.M. Sandalio, Peroxisome dynamics in Arabidopsis plants under oxidative stress induced by cadmium, Free Radic. Biol. Med. 47 (2009) 1632-1639.

[36] J.E. Legakis, J.I. Koepke, C. Jedeszko, F. Barlaskar, L.J. Terlecky, H.J. Edwards, P.A. Walton, S.R. Terlecky, Peroxisome senescence in human fibroblasts, Mol. Biol. Cell 13 (2002) 4243-4255.

[37] K. Murakami, Y. Ichinohe, M. Koike, N. Sasaoka, S. Iemura, T. Natsume, A. Kakizuka, VCP is an integral component of a novel feedback mechanism that controls intracellular localization of catalase and $\mathrm{H}_{2} \mathrm{O}_{2}$ levels, PLoS ONE 8 (2013) e56012.

[38] V.E. Pye, C.E. Christensen, J.H. Dyer, S. Arent, A. Henriksen, Peroxisomal plant 3ketoacyl-CoA thiolase structure and activity are regulated by a sensitive redox switch, J. Biol. Chem. 285 (2010) 24078-27088.

[39] L.A. del Río, Peroxisomes as a cellular source of reactive nitrogen species signal molecules, Arch. Biochem. Biophys. 506 (2011) 1-11. 
[40] A. Beach, M.T. Burstein, V.R. Richard, A. Leonov, S. Levy, V.I. Titorenko, Integration of peroxisomes into an endomembrane system that governs cellular aging, Front. Physiol. 3 (2012). doi: 10.3389/fphys.2012.00283.

[41] Z.T. Schug, C. Frezza, L.C. Galbraith, E. Gottlieb, The music of lipids: how lipid composition orchestrates cellular behavior, Acta Oncol. 51 (2012) 301-310.

[42] B.T. Bikman, S.A. Summers, Ceramides as modulators of cellular and whole-body metabolism, J. Clin. Invest. 121 (2011) 4222-4230.

[43] S. Hekimi, J. Lapointe, Y. Wen, Taking a "good" look at free radicals in the aging process, Trends Cell Biol. 21 (2011) 569-576.

[44] A.B. Fisher, Redox signaling across cell membranes, Antioxid. Redox Signal. 11 (2009) 1349-1356.

[45] H.J. Forman, M. Maiorino, F. Ursini, Signaling functions of reactive oxygen species, Biochemistry 49 (2010) 835-842.

[46] D. Fass, Hunting for alternative disulfide bond formation pathways: endoplasmic reticulum janitor turns professor and teaches a lesson, Mol. Cell. 40 (2010) 685-686.

[47] H. Wang, M. Xian, Chemical methods to detect S-nitrosation. Curr. Opin. Chem. Biol. 15 (2011) 32-37.

[48] Y. Yang, Y. Song, J. Loscalzo, Regulation of the protein disulfide proteome by mitochondria in mammalian cells, Proc. Natl. Acad. Sci. U.S.A. 104 (2007) 1081310817.

[49] X. Chen, H. Liang, H. Van Remmen, J. Vijg, A. Richardson, Catalase transgenic mice: characterization and sensitivity to oxidative stress, Arch. Biochem. Biophys. 422 (2004) 197-210.

[50] A.B. Carter, L.A. Tephly, S. Venkataraman, L.W. Oberley, Y. Zhang, G.R. Buettner, D.R. Spitz, G.W. Hunninghake, High levels of catalase and glutathione peroxidase activity dampen $\mathrm{H}_{2} \mathrm{O}_{2}$ signaling in human alveolar macrophages, Am. J. Respir. Cell Mol. Biol. 31 (2004) 43-53.

[51] Y. Li, J.C. Tharappel, S. Cooper, M. Glenn, H.P. Glauert, B.T. Spear, Expression of the hydrogen peroxide-generating enzyme fatty acyl-CoA oxidase activates NF-kappaB, DNA Cell Biol. 19 (2000) 113-120.

[52] M. Elsner, W. Gehrmann, S. Lenzen, Peroxisome-generated hydrogen peroxide as important mediator of lipotoxicity in insulin-producing cells, Diabetes 60 (2011) 200208. 
[53] W.M. Frederiks, H. Vreeling-Sindelárová, Ultrastructural localization of xanthine oxidoreductase activity in isolated rat liver cells, Acta Histochem. 104 (2002) 29-37.

[54] D.B. Stolz, R. Zamora, Y. Vodovotz, P.A. Loughran, T.R. Billiar, Y.M. Kim, R.L. Simmons, S.C. Watkins, Peroxisomal localization of inducible nitric oxide synthase in hepatocytes, Hepatology 36 (2002) 81-93.

[55] P.T. Doulias, M. Tenopoulou, J.L. Greene, K. Raju, H. Ischiropoulos, Nitric oxide regulates mitochondrial fatty acid metabolism through reversible protein $\mathrm{S}$ nitrosylation, Sci. Signal. 6 (2013). doi: 10.1126/scisignal.2003252.

[56] P.A. Loughran, D.B. Stolz, Y. Vodovotz, S.C. Watkins, R.L. Simmons, T.R. Billiar, Monomeric inducible nitric oxide synthase localizes to peroxisomes in hepatocytes, Proc. Natl. Acad. Sci. U.S.A. 102 (2005) 13837-13842.

[57] L. Liaudet, G. Vassalli, P. Pacher, Role of peroxynitrite in the redox regulation of cell signal transduction pathways, Front. Biosci. 14 (2009) 4809-4814.

[58] N.E. Braverman, A.B. Moser, Functions of plasmalogen lipids in health and disease, Biochim. Biophys. Acta. 1822 (2012) 1442-1452.

[59] K.S. Fritz, D.R. Petersen, An overview of the chemistry and biology of reactive aldehydes, Free Radic. Biol. Med. 59 (2013) 85-91.

[60] Z.H. Chen, E. Niki, 4-hydroxynonenal (4-HNE) has been widely accepted as an inducer of oxidative stress. Is this the whole truth about it or can 4-HNE also exert protective effects? IUBMB Life 58 (2006) 372-373.

[61] B.J. Pettus, M. Baes, M. Busman, Y.A. Hannun, P.P. Van Veldhoven, Mass spectrometric analysis of ceramide perturbations in brain and fibroblasts of mice and human patients with peroxisomal disorders, Rapid Commun. Mass Spectrom. 18 (2004) 1569-1574.

[62] M. Schrader, Y. Yoon, Mitochondria and peroxisomes: are the 'big brother' and the 'little sister' closer than assumed? Bioessays 29 (2007) 1105-1114.

[63] E. Dixit, S. Boulant, Y. Zhang, A.S. Lee, C. Odendall, B. Shum, N. Hacohen, Z.J. Chen, S.P. Whelan, M. Fransen, M.L. Nibert, G. Superti-Furga, J.C. Kagan, Peroxisomes are signaling platforms for antiviral innate immunity, Cell 141 (2010) 668-681.

[64] S.D. Kohlwein, M. Veenhuis, I.J. van der Klei, Lipid droplets and peroxisomes: key players in cellular lipid homeostasis or a matter of fat - store 'em up or burn 'em down, Genetics 193 (2013) 1-50.

[65] J. Koch, C. Brocard, PEX11 proteins attract Mff and human Fis1 to coordinate peroxisomal fission, J. Cell Sci. 125 (2012) 3813-3826. 
[66] M. Schrader, N.A. Bonekamp, M. Islinger, Fission and proliferation of peroxisomes, Biochim. Biophys. Acta 1822 (2012) 1343-1357.

[67] M. Ott, V. Gogvadze, S. Orrenius, B. Zhivotovsky, Mitochondria, oxidative stress and cell death, Apoptosis 12 (2007) 913-922.

[68] E. Baumgart, I. Vanhorebeek, M. Grabenbauer, M. Borgers, P.E. Declercq, H.D. Fahimi, M. Baes, Mitochondrial alterations caused by defective peroxisomal biogenesis in a mouse model for Zellweger syndrome (PEX5 knockout mouse), Am. J. Pathol. 159 (2001) 1477-1494.

[69] R. Dirkx, I. Vanhorebeek, K. Martens, A. Schad, M. Grabenbauer, D. Fahimi, P. Declercq, P.P. Van Veldhoven, M. Baes, Absence of peroxisomes in mouse hepatocytes causes mitochondrial and ER abnormalities, Hepatology 41 (2005) 868-878.

[70] J. López-Erauskin, J. Galino, M. Ruiz, J.M. Cuezva, I. Fabregat, D. Cacabelos, J. Boada, J. Martínez, I. Ferrer, R. Pamplona, F. Villarroya, M. Portero-Otín, S. Fourcade, A. Pujol, Impaired mitochondrial oxidative phosphorylation in the peroxisomal disease X-linked adrenoleukodystrophy, Hum. Mol. Genet. (2013). In press.

[71] Y.S. Ho, Y. Xiong, W. Ma, A. Spector, D.S. Ho, Mice lacking catalase develop normally but show differential sensitivity to oxidant tissue injury, J. Biol. Chem. 279 (2004) 32804-32812.

[72] I. Hwang, J. Lee, J.Y. Huh, J. Park, H.B. Lee, Y.S. Ho, H. Ha, Catalase deficiency accelerates diabetic renal injury through peroxisomal dysfunction, Diabetes 61 (2012) 728-738.

[73] V.I. Titorenko, S.R. Terlecky, Peroxisome metabolism and cellular aging, Traffic 12 (2011) 252-259.

[74] K. Hasegawa, S. Wakino, K. Yoshioka, S. Tatematsu, Y. Hara, H. Minakuchi, K. Sueyasu, N. Washida, H. Tokuyama, M. Tzukerman, K. Skorecki, K. Hayashi, H. Itoh, Kidney-specific overexpression of Sirt1 protects against acute kidney injury by retaining peroxisome function, J. Biol. Chem. 285 (2010) 13045-13056.

[75] S. Diano, Z.W. Liu, J.K. Jeong, M.O. Dietrich, H.B. Ruan, E. Kim, S. Suyama, K. Kelly, E. Gyengesi, J.L. Arbiser, D.D. Belsham, D.A. Sarruf, M.W. Schwartz, A.M. Bennett, M. Shanabrough, C.V. Mobbs, X. Yang, X.B. Gao, T.L. Horvath, Peroxisome proliferation-associated control of reactive oxygen species sets melanocortin tone and feeding in diet-induced obesity, Nat. Med. 17 (2011) 1121-1127. 
[76] J. López-Erauskin, J. Galino, P. Bianchi, S. Fourcade, A.L. Andreu, I. Ferrer, C. Muñoz-Pinedo, A. Pujol, Oxidative stress modulates mitochondrial failure and cyclophilin D function in X-linked adrenoleukodystrophy, Brain 135 (2012) 3584-3598.

[77] S. Gandhi, A.Y. Abramov, Mechanism of oxidative stress in neurodegeneration, Oxid. Med. Cell. Longev. (2012). doi: 10.1155/2012/428010.

[78] G. Lizard, O. Rouaud, J. Demarquoy, M. Cherkaoui-Malki, L. Iuliano, Potential roles of peroxisomes in Alzheimer's disease and in dementia of the Alzheimer's type, J. Alzheimers Dis. 29 (2012) 241-254.

[79] K. Beier, A. Völkl, H.D. Fahimi, The impact of aging on enzyme proteins of rat liver peroxisomes: quantitative analysis by immunoblotting and immunoelectron microscopy, Virchows Arch. B. Cell Pathol. Incl. Mol. Pathol. 63 (1993) 139-146.

[80] C. Chao, J. Youssef, M. Rezaiekhaleigh, L.S. Birnbaum, M. Badr, Senescenceassociated decline in hepatic peroxisomal enzyme activities corresponds with diminished levels of retinoid X receptor alpha, but not peroxisome proliferator-activated receptor alpha, Mech. Ageing Dev. 123 (2002) 1469-1476.

[81] C.S. Wood, J.I. Koepke, H. Teng, K.K. Boucher, S. Katz, P. Chang, L.J. Terlecky, I. Papanayotou, P.A. Walton, S.R. Terlecky, Hypocatalasemic fibroblasts accumulate hydrogen peroxide and display age-associated pathologies, Traffic 7 (2006) 97-107.

[82] F. Fanelli, S. Sepe, M. D'Amelio, C. Bernardi, L. Cristiano, A. Cimini, F. Cecconi, M.P. Ceru', S. Moreno, Age-dependent roles of peroxisomes in the hippocampus of a transgenic mouse model of Alzheimer's disease, Mol. Neurodegener. 8 (2013) 8. doi: $10.1186 / 1750-1326-8-8$.

[83] D.J. Baker, T. Wijshake, T. Tchkonia, N.K. LeBrasseur, B.G. Childs, B. van de Sluis, J.L. Kirkland, J.M. van Deursen, Clearance of p16Ink4a-positive senescent cells delays ageing-associated disorders, Nature 479 (2011) 232-236.

[84] S.R. Terlecky, L.J. Terlecky, C.R. Giordano, Peroxisomes, oxidative stress, and inflammation, World J. Biol. Chem. 3 (2012) 93-97.

[85] I. Singh, A.K. Singh, M.A. Contreras, Peroxisomal dysfunction in inflammatory childhood white matter disorders: an unexpected contributor to neuropathology, J. Child Neurol. 24 (2009) 1147-1157.

[86] J.W. Zmijewski, E. Lorne, X. Zhao, Y. Tsuruta, Y. Sha, G. Liu, E. Abraham, Antiinflammatory effects of hydrogen peroxide in neutrophil activation and acute lung injury, Am. J. Respir. Crit. Care Med. 179 (2009) 694-704. 
[87] H.I. El Hajj, A. Vluggens, P. Andreoletti, K. Ragot, S. Mandard, S. Kersten, H.R. Waterham, G. Lizard, R.J. Wanders, J.K. Reddy, M. Cherkaoui-Malki, The inflammatory response in acyl-CoA oxidase 1 deficiency (pseudoneonatal adrenoleukodystrophy), Endocrinology 153 (2012) 2568-2575.

[88] A. Bottelbergs, S. Verheijden, P.P. Van Veldhoven, W. Just, R. Devos, M. Baes, Peroxisome deficiency but not the defect in ether lipid synthesis causes activation of the innate immune system and axonal loss in the central nervous system, J. Neuroinflammation 9 (2012) doi: 10.1186/1742-2094-9-61.

[89] M. Khan, K. Pahan, A.K. Singh, I. Singh, Cytokine-induced accumulation of very longchain fatty acids in rat C6 glial cells: implication for X-adrenoleukodystrophy, J. Neurochem. 71 (1998) 78-87. 


\section{Figure caption}

Fig. 1. Hypothetical model depicting the role of peroxisomes in cell fate decisions. Peroxisomes play a central role in cellular lipid and ROS/RNS metabolism. As such, the metabolic output of these organelles can affect mitochondrial function and modulate the bioavailability of lipid- and redox-related factors involved in diverse cellular signaling pathways. Depending on the specific pathways affected, these changes exert either cytoprotective or cytotoxic actions. The phenomenon in which peroxisome function is adapted to exert beneficial effects (e.g. to protect cells against oxidative insults, to promote cell survival and proliferation pathways, ...), is called 'peroxihormesis'. Disturbances in peroxisome function (e.g. upon acute or chronic inflammation and/or exposure to oxidative stress) can evoke oxidative stress-mediated signaling mechanisms associated with cellular aging and age-related diseases. PUFAs, polyunsaturated fatty acids; RNS, reactive nitrogen species; ROS, reactive oxygen species; VLCFAs, very-long-chain fatty acids. 


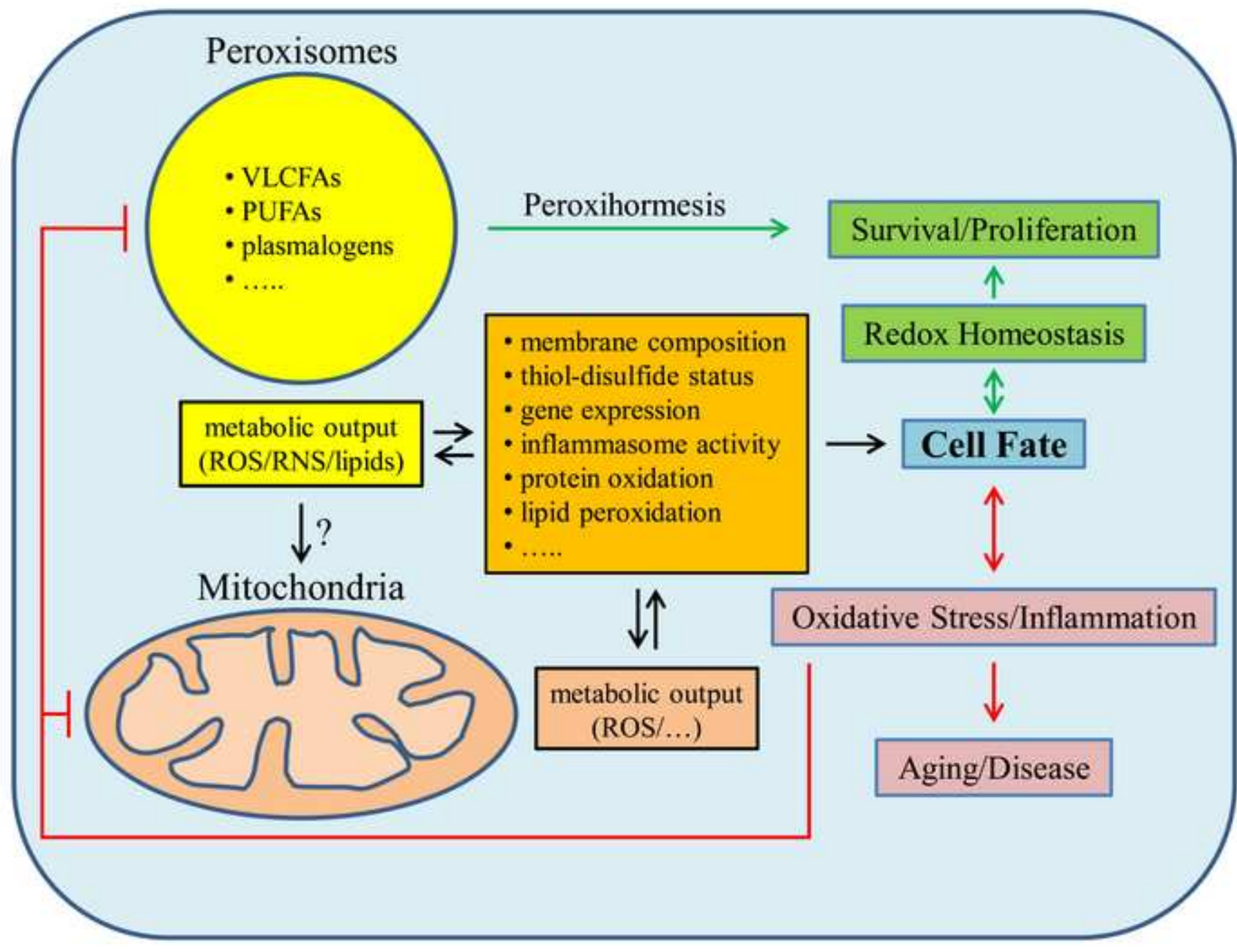

